\title{
The use of DCEEG to estimate functional and metabolic state of nervous tissue of the brain at hyper- and hypoventilation
}

\author{
Sergey Murik \\ Department of Physiology and Psychophysiology, Irkutsk State University, Irkutsk, Russia \\ Email: sergey_murik@yahoo.com
}

Received 21 November 2011; revised 23 December 2011; accepted 17 January 2012

\begin{abstract}
A pilot study has been made of the simultaneous DC potential and total slow electrical activity changes during modeling various metabolic and functional states of the human brain. The multi-electrode DCEEG recordings have been performed during the hyperventilation (frequent deep one-minute long breathing motions) and the hypoventilation (voluntary breath holding). It has been shown that the ischemic state occurring in hyperventilation is accompanied by the negative shift of DC potential and increase in the EEG rhythms amplitude. A distention of brain vessels during hypoventilation (voluntary breath-hold) and an improvement of blood supply and thus improvement of vital and functional state of neurons gave rise to an increase in the EEG rhythm amplitude too, though against a background of a positive DC-potential shift. Obtained results are considered with context the generation of the qualitatively different functional states of brain cells during hyper- and hypoventilation which is reflected in their resting potential and activity. The conducted study show the prospects for DCEEG and the method we used for DCEEG data processing to understand the character of functional and metabolic changes in the nervous tissue.
\end{abstract}

Keywords: DCEEG; Full-Band EEG (fbEEG); Functional State of the Nervous Tissue; Hyperventilation; Hypoventilation; Breath Holding; Ischemia

\section{INTRODUCTION}

In the first half of the last decade, there appeared a number of publications $[1,2]$ considering full-band EEG (fbEEG) as a promising method that could succeed the classical EEG. FbEEG implies macro-electrode recording of brain potentials in a wide frequency range from 0 to $100 \mathrm{~Hz}$ and higher. This approach may be realized using the DC amplifier and recording the classical EEG rhythms simultaneously with the DC potential and its low-frequency $(<1 \mathrm{~Hz})$ but often high-amplitude (about 1 millivolt and higher) oscillations.

The method of simultaneous recording of DC potential and EEG can be called the direct current EEG (DCEEG) too. However, very few electrophysiological studies using this method have been reported in recent years. This can be partly attributed to the fact that the nature of DC potential is still unclear. Numerous works published before the late 1990s implied the neuronal nature of DC potential [3-10]. The negative DC potential shift was considered as a reflection of depolarization processes in the neuroglial complex [4,5,11-17]. Such understanding implied that the positive DC potential shift was responsible for re- or hyperpolarization of nervous tissue cells occurring under an active electrode.

However, in the early 21 century some publications $[18,19]$ appeared concerning one of the old ideas [20] about the possibility of non-neuronal origin of the DC potential. This idea implies that the DC potential recorded from the brain surface and the scalp is the potential difference occurring at the boundary between the arterial and venous circulatory systems, determined by ion gradients between arterial and venous blood and interstitial fluid accumulated in brain ventricles. The basic argument for this concept made by its supporters is the identified DC potential change along with vascular responses and alteration of the $\mathrm{CO}_{2}$ and $\mathrm{O}_{2}$ contents of the blood $[18,19]$. The authors reported that the rise or fall of these gases in blood cause the DC potential shift. They consider this relationship a direct indication for the nonneuronal nature of the DC potential. However, the fact that the change in concentration of these gases and vascular responses may cause changes in metabolism in the neurons and thus shifts in polarization of their membrane is neither considered nor studied experimentally by the authors.

We suppose that the number of arguments supplied for non-neuronal origin of the DC potential is insufficient to 
deny the arguments and facts obtained over many decades by tens of investigators in the support of the neuronal nature of this potential.

Earlier, we developed [21], an animal-experiment-based way to record and process DCEEG wherein the character of change in the EEG rhythm amplitudes in any lead was simultaneously compared to the character of DC potential shifts. Analysis of simultaneous changes in the EEG and DC potential provided an opportunity to reveal the features of the functional and metabolic state of nervous tissue when modeling a number of effects on an animal organism that could not be detected with other electrophysiological methods. This method has not yet been applied to human beings.

The aim of this work is to estimate whether simultaneous recording of DC potential and EEG by the method of Murik, S.E. (2004) and Murik, S.E. and Shapkin, A.G. (2004) could be applied to human beings.

DCEEG recording for a human being is associated with a number of problems: the influence of the so-called skin potentials and the drift of electrode potentials [2224]. Therefore, one of the tasks was to estimate the possibility and complexity of simultaneous multi-electrode recording of DC and EEG from human scalp. For models of different functional state (FS) we chose functional tests: hyperventilation $[14,25,26]$ and hypoventilation $[27,28]$.

\section{MATERIALS AND METHODS}

Until recently, the brain DC potential was usually recorded separately from classical EEG and its recording technique was reasonably developed [2,23,25]. For simultaneous recording of the brain DC potential and EEG, we have used the technique somewhat modified relative to the DC potential recording techniques already reported. A characteristic of the DC potential recording is the necessity to remove electrode and skin potentials [22-24]. The electrode potentials and their drifts can be removed with the use of non-polarizing electrodes, selection of optimal EEG gel, and special electrode preparation that involves soaking the electrodes in the EEG gel for no less than $15 \mathrm{~min}$. The skin potentials can be removed with the use of special treatment for the scalp that often includes peeling the epidermis with a needle or anesthesia, and defatting and tanning the scalp [14,23,25]. Scalp preparation for electrode placement causes certain difficulties and is unpleasant for subjects. That may be the reason no more than 5 - 6 leads have so far been used for recording of the DC potential. Using DCEEG in clinics requires recording the electrical activity at least via 16 channels and an easier and less painful scalp preparation for electrode placement. Therefore, the technique development has involved the search of way to perform DCEEG multi-channel recording that would be easier on the one hand and provide the possibility for satisfactory recording DC potential on the other. Such way seems to have been found and is reported below.

\subsection{Subjects}

Our experiments involved 6 healthy subjects ( 2 men and 4 women between the age of 22 and 51, average age of $29 \pm 5.4$ years) five of which were between 22 and 27 years old, much younger than the 51-year-old subject. However, the individual analysis has shown that the results obtained for the 51-year-old subject and for the younger subjects are quantitatively similar and thus may be combined.

All subjects were informed about all aspects of the experiments, and all gave an informed consent. We conformed to the Declaration of Helsinki.

\subsection{DCEEG Recording Technique}

The DCEEG recording has been performed using the unipolar technique by the computer electroencephalograph with the 64-channel DC amplifier designed by ANT Company (asa-lab EEG system, Netherlands). The DCEEG data without low-frequency limit have been digitized at the $512 \mathrm{~Hz}$ frequency. The indifferent (reference) electrodes were stuck on the ear lobes. The DCEEG recording has been performed using standard $\mathrm{Ag} / \mathrm{AgCl}$ electrodes built in the EEG cap supplied together with the EEG system by the manufacturing company (ANT, Netherlands). The plastic holders of the recording electrodes were filled with thick EEG paste (Ten20, Weaver and Co., USA) an hour before putting an EEG cap on. The DCEEG has been recorded from 38 electrodes placed at Fp1, Fp2, Fz, F3, F4, F7, F8, FCz, FC1, FC2, FC3, FC4, FC5, FC6, Cz, C1, C2, C3, C4, C5, C6, T7, T8, CPz, CP1, CP2, CP3, CP4, CP5, CP6, Pz, P3, P4, P7, P8, Oz, O1, O2.

Putting a cap on was preceded by thorough treatment of the whole scalp and ear lobes of the subjects with abrasive gel (Abralyt 2000, EASYCAP GmbH, Germany). Thereafter the head was washed with shampoo and toweled dry, and the scalp and hair were covered with a thin layer of the same EEG paste that was used for the electrodes (Ten20, Weaver and Co., USA). After the EEG paste treatment, a cap was put on the head, indifferent electrodes were stuck on the ear lobes, and EOG electrodes were stuck on the face. An impedance test as applied to the proposed technique of the preparation of the head and EEG cap has shown that the EEG electrode impedance was much lower than $3 \mathrm{kOhm}$ and remained unchanged until the end of the experiment.

The DCEEG recording began immediately after the impedance measurement and was performed throughout the entire multi-stage experiment that lasted approxi- 
mately 2 hours. During the first 1 -hour stage of resting state the subject had to accommodate himself to the general conditions of the experiment. A long duration of the first stage was also required for reduction of probable electrode and skin potentials.

Throughout the entire experiment, the subject was in an EEG armchair, with his head rested on the head holder. Before the beginning of the EEG recording, the subject chose the most comfortable position of the head holder and armchair back. In the first $10-15$ minutes of the resting-state stage, the subject got the instruction for the experimental procedure and his behavior at various experimental stages. The instruction particularly emphasized that during a major part of experiment, including the functional tests, the subject should neither talk nor move any of his limbs, head or body. He was only allowed to speak if he felt bad. During a major part of the experiment whose start would be reported, he should only look at the target without any other eye movements. A target shaped in the form of a $4 \times 4$ cross, drawn on a A4 sheet, was placed on the wall $2 \mathrm{~m}$ in front of the subject before the beginning of the DCEEG recording. The instruction also reminded the subject of the contents of functional tests that had been detailed since before the start of the entire experiment.

\subsection{Functional Tests}

In a major part of the experiment, the subject did two functional tests-hyper- and hypoventilation-successively at the experimentalist's command. The testing succession varied from one subject to another. The testing interval was $20 \mathrm{~min}$.

During the hyperventilation test, the subject had to begin making frequent deep one-minute long breathing motions at the experimentalist's command. The subject had 30 deep breathing cycles in one minute. The breathing frequency was set by the breath in/breath out experimentalist's commands. The start of the hyperventilation test was preceded by the experimentalist's phrase: "Let's start the hyperventilation test, take a deep breath and breathe out deeply at the experimentalist's command. We start!"

The hypoventilation test implied the subject breath holding for as long as possible after the quiet inhale. The start of the hypoventilation test was preceded by the experimentalist's phrase: "Let's start the hypoventilation test, inhale quietly and hold your breath for as long as possible. We start!"

\subsection{DCEEG Data Processing}

The processing was performed with the use of native DCEEG containing the DC potential and its fluctuations in the range from 0 to $205 \mathrm{~Hz}$. For obtaining the data on
EEG rhythm amplitude and DC potential level, the entire DCEEG was primarily divided into one-second intervals using Brain Vision Analyzer software (Brain Products $\mathrm{GmbH}$, Germany), after which a four-range rhythm capacity spectrum has been obtained using fast Fourier transform: delta $(0.5-4 \mathrm{~Hz})$, theta $(4-8 \mathrm{~Hz})$, alpha (8 $12 \mathrm{~Hz})$ and beta $(12-30 \mathrm{~Hz})$. Then the DC potential level was averaged over the same time intervals using a special program. Such primary processing of the initial DCEEG over every second of time provided the possibility to obtain the data on both EEG rhythm amplitudes in four ranges and DC potential level.

For the further processing, the segments related to the functional tests were cut off from such complex DCEEG. The hyperventilation test analysis has been made using a continuous segment with a three-minute period before the test start, a one-minute period of hyperventilation, and a ten-minute period that followed after the test finish. The hypoventilation data analysis involved a continuous segment with a three-minute period before the test start, the period of hypoventilation, and the period immediately after the hypoventilation test finish that took $6 \mathrm{~min}$ together with the period of hyperventilation. The duration of hypoventilation period might vary due to personal peculiarities of the subjects.

\subsection{Data Analysis}

For estimating the functional test influence on the DC potential and EEG amplitude, the every-second DC potential levels and EEG rhythm amplitudes were averaged over $30 \mathrm{~s}$ and 1 minute before the start of functional tests of hyper- and hypoventilation. The obtained level was taken for the initial one to be compared to the 1-second DC potential levels and 30-second or 1-minute EEG rhythm amplitudes during and after hyper- and hypoventilation. The DC potential and EEG rhythm amplitude were sometimes averaged over other time intervals.

The data processing was primarily performed for some of the leads, after which the results were averaged over all the leads for some of the subjects and, finally, for all of the subjects. The mathematical and statistical processing of the data has been performed with Microsoft Office Excel. The significance of differences in the data has been determined using the Student's $t$-test for dependent and independent samplings.

\section{RESULTS}

\subsection{Hyperventilation}

Even relatively long-term (one-hour) presence of EEG electrode paste before the cap is put on requires yet another 15 - 30 minutes after it has been put on to remove the DC potential drifts. Our observation shows that the 
optimum time to reduce the drift of the DC potential is 30 min. Our pilot study has also shown that the DC potential may be either recorded without peeling the epidermis by a needle [23]. Generally, it is enough to massage the scalp thoroughly with the fingertips using abrasive gel. At least, our finding of the DC potential behavior during hyperventilation generally corresponds to that obtained during mechanical peeling of the scalp with a needle and other treatments [14,23,25]. Figure 1 shows the mean levels $(n=6)$ of DC potential and EEG amplitude change in four ranges before $(3 \mathrm{~min})$, during (1 $\mathrm{min}$ ) and after (10 min) hyperventilation, averaged over all the leads. It is seen that hyperventilation has caused a negative shift of the DC potential (for about $400 \pm 86 \mu \mathrm{V}, \mathrm{p} \leq$ $0.001)$ and an increase of EEG rhythm amplitudes in the delta (for $6.3 \%, p<0.05$, Table 1) and beta (for $17.6 \%$, $\mathrm{p} \leq 0.001)$ ranges.

However, the negative DC potential shift (negativation of the DC potential) more often than not continued sometime after hyperventilation and attained its maximum $(461.2 \pm 78 \mu \mathrm{V})$ in 5 - 20 seconds, with the rhythm amplitudes increased not only in the delta and beta ranges but also at theta and alpha frequencies. During the first 30 seconds after hyperventilation test, the EEG rhythm amplitudes were generally higher than during $30 \mathrm{sec}$ be- fore hyperventilation: by $27.5 \%(\mathrm{p} \leq 0.001)$ in delta range, $18.9 \%(\mathrm{p} \leq 0.001)$ in theta range, $27.6 \%(\mathrm{p} \leq$ $0.001)$ in alpha range and $30.8 \%(\mathrm{p} \leq 0.001)$ in beta range. After 1-minute hyperventilation had been stopped, the rate of recovery (positivation) of the DC potential was lower than that of its negativation and lasted about 4 minutes.

The study of changes in the DC potential during hyperventilation applied to separate leads has shown that the amplitude of the negative DC potential shift could differ significantly in front (Fz, F3, F4, F7, F8, FCz, FC1, FC2, FC3, FC4, FC5, FC6), central (Cz, C1, C2, C3, C4, C5,C6, T7, T8) and back (CPz, CP1, CP2, CP3, CP4, $\mathrm{CP} 5, \mathrm{CP} 6, \mathrm{Pz}, \mathrm{P} 3, \mathrm{P} 4, \mathrm{P} 7, \mathrm{P} 8, \mathrm{Oz}, \mathrm{O} 1, \mathrm{O} 2)$ areas. The largest negative DC potential shift occurred in front leads and was, on average, $428.5 \pm 20.2 \mu \mathrm{V}$. Negativation of the DC potential in the central and back leads was, on average, $402.0 \pm 24.5 \mu \mathrm{V}$ and $345.6 \pm 26.2 \mu \mathrm{V}$, respectively. The difference in the negative DC potential shift between the front and back areas was significant ( $\mathrm{p} \leq$ 0.01 ). Therefore, it is obvious that there is a gradient for the DC potential response to hyperventilation: the negative DC potential shift occurred mostly in the front areas of neocortex, to a lesser extent - in the central, and minimum - in the back areas.

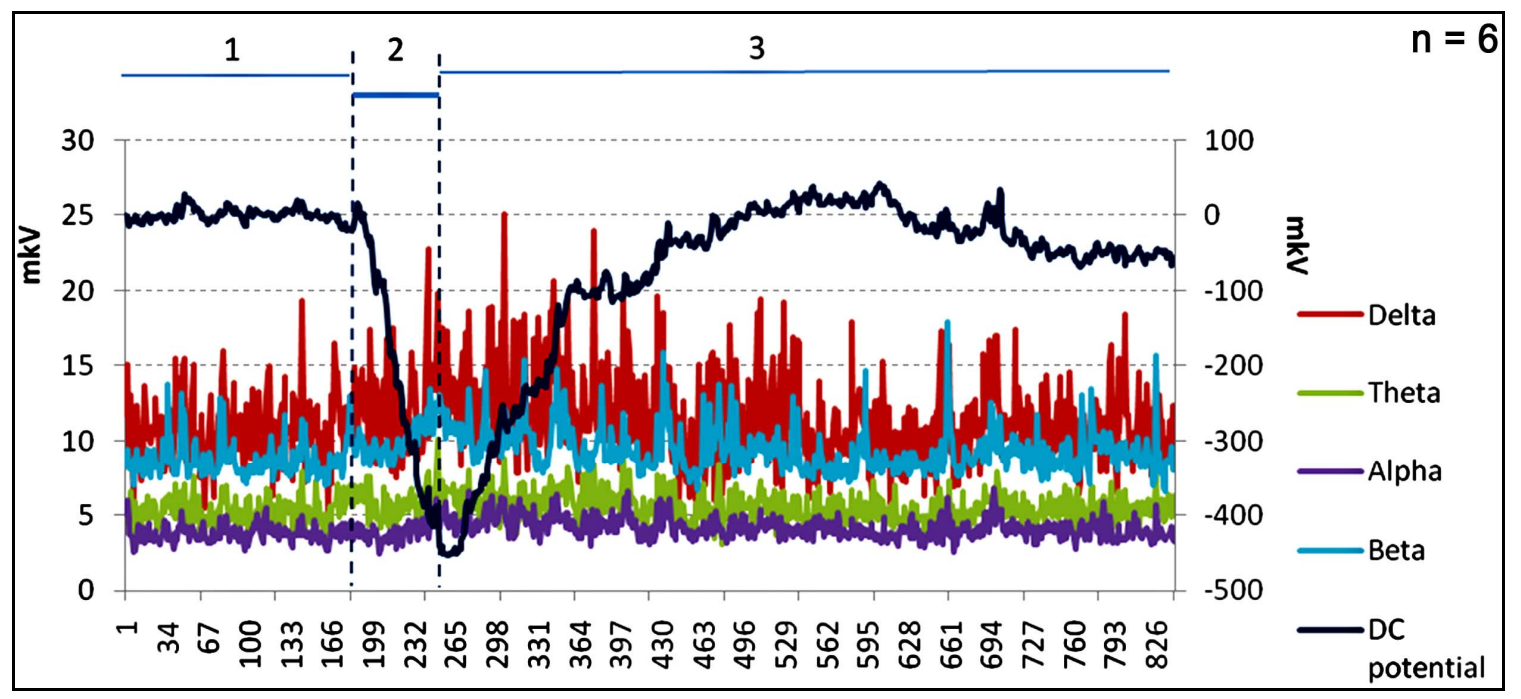

Figure 1. Simultaneous changes in the DC potential and four-range EEG amplitudes before (1), during (2) and after (3) hyperventilation for the group as a whole $(n=6)$. The averaging has been made over all the leads $(n=38)$. The left Y-axis represents the amplitude of EEG rhythms $(\mathrm{mkV})$, and the right Y-axis represents that of the DC potential $(\mathrm{mkV})$.

Table 1. The level of EEG rhythm amplitudes $(\mu \mathrm{V}, \mathrm{m} \pm \mathrm{SEM})$ over $30 \mathrm{sec}$ before, during and immediately after hyperventilation $(\mathrm{n}=6)$. Designations: ${ }^{*} \mathrm{p}<0.05 ;{ }^{* * *} \mathrm{p} \leq 0.001$ as compared to the level before hyperventilation.

\begin{tabular}{lllll}
\hline & Delta & Theta & Alpha & Beta \\
\hline 30 s before hyperventilation & $10.9 \pm 0.3$ & $5.5 \pm 0.2$ & $3.7 \pm 0.1$ & $8.6 \pm 0.3$ \\
30 s before the end of hyperventilation & $11.6 \pm 0.6^{*}$ & $5.5 \pm 0.2$ & $3.9 \pm 0.2$ & $10.1 \pm 0.4^{* * *}$ \\
30 s immediately after hyperventilation & $13.9 \pm 8.4^{* * *}$ & $6.5 \pm 0.2^{* * *}$ & $4.7 \pm 0.2^{* * *}$ & $11.3 \pm 0.3^{* * *}$ \\
\hline
\end{tabular}


The differences in negativation of the DC potential during hyperventilation have been also found from interhemispheric comparison. The DC potential shift in the left hemisphere was larger than that in the right and reached, on average, $404.0 \pm 15.5 \mu \mathrm{V}$ and $357.9 \pm 19.5$ $\mu \mathrm{V}(\mathrm{p}<0.05)$, respectively.

Therefore, the averaging of DCEEG over all the leads $(n=38)$ and subjects $(n=6)$ during hyperventilation was associated with the negative DC potential shift and EEG rhythm capacity increase. Generally, these results corresponded to those already available that were obtained during an independent recording of DC potential and EEG [14]. However, individual analysis of DCEEG showed some individual peculiarities of simultaneous changes of the DC potential and EEG that had not been found so far. Basic types of simultaneous changes of DCEEG are shown in Figure 2. As evident from the figure, the differences in the DC potential were mainly associated with the character of its recovery after hyperventilation test had been stopped. There might be both delay in recovery of the initial DC potential level (subject No. 6) and posthyperventilation positive DC wave (subject No. 4 and subject No. 5). Therefore, the individual peculiarities of DCEEG are longer or shorter delay in recovery of the initial DC potential level and presence or absence of posthyperventilation positive wave.
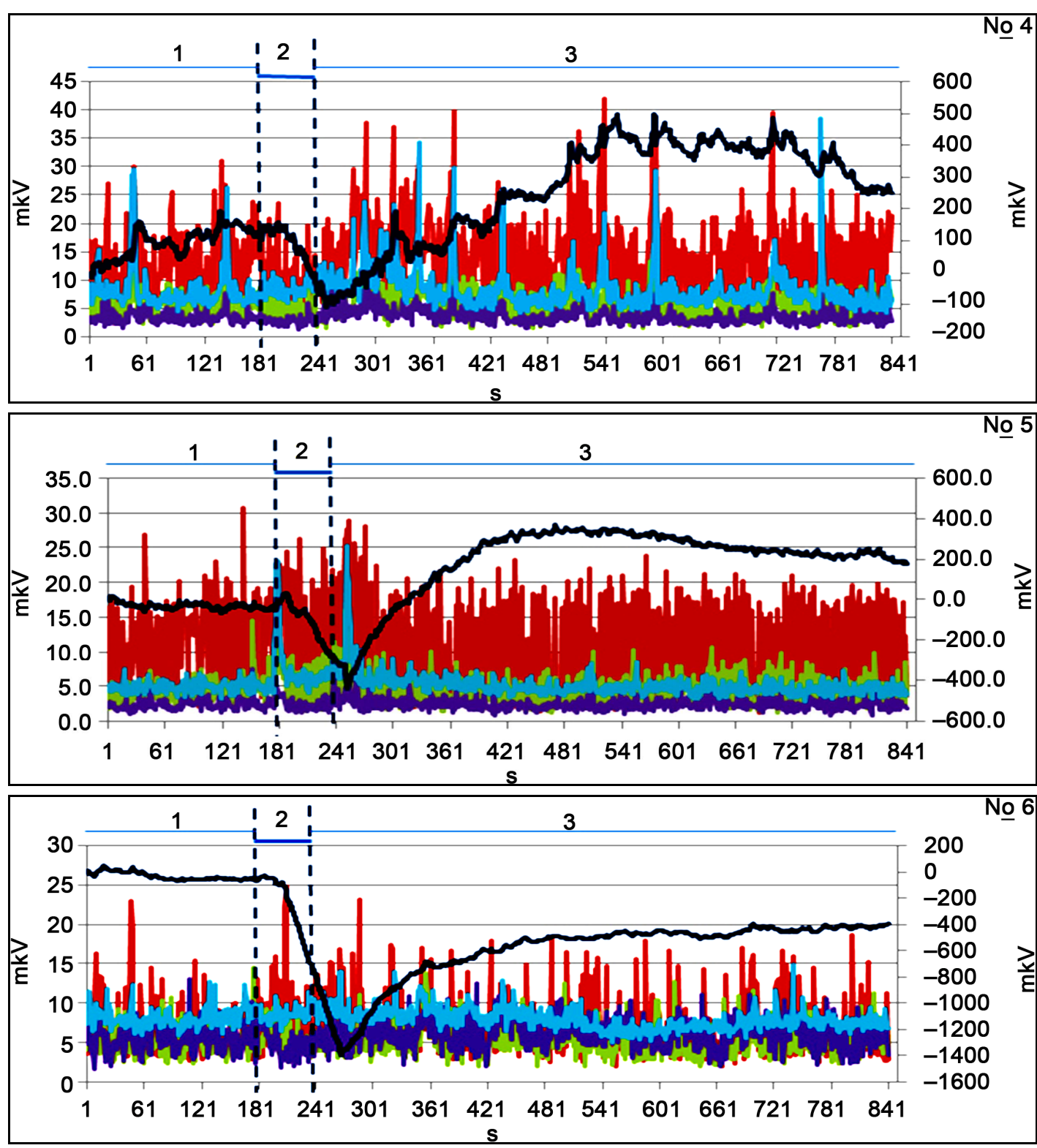

Figure 2. Individual peculiarities of simultaneous changes in DC potential and EEG of three subjects (No. 4, No. 5 and No. 6). The averaging has been performed over all the leads for each subject. The designations are the same as in Figure 1. 
DCEEG analysis on separate leads has shown a yet larger variability in the character of simultaneous changes in the DC potential and EEG (see Figure 3). As may be seen from this figure, negativation of the DC potential during hyperventilation may be preceded by the phase of positivation of the DC potential (Fp1). The phase of positivation (the positive DC potential shift) may sometimes extend and take almost all hyperventilation time (FC1).

\subsection{Hypoventilation}

A pylot study of DCEEG associated with breath-holding spells has also shown significant simultaneous changes in the DC potential and EEG that occurred both during and after the test. There are certain problems with direct superposition and averaging of the DC potential and the data on EEG amplitude change obtained after the breathholding because the breath-holding duration was characterized by the individual data scattering in the wide 33 $104 \mathrm{sec}$ range $(56.0 \pm 22.9$ on the average). Individual simultaneous changes in the DC potential and EEG in separate leads are shown for three subjects in Figure 4.

A specific feature of the DCEEGF variation during hypoventilation was that the breath-hold generally caused the positive DC potential shift and an ambiguous change in the EEG rhythm amplitudes at that time. By the time of breath-holding termination, the positive DC potential shift was, on average, $314.3 \pm 64.9 \mu \mathrm{V}$ ( $\mathrm{p}<$ $0.01)$ for the whole group of the subjects. The rhythm amplitude at that time only increased in the beta-range$26.9 \%(\mathrm{p}<0.001)$ (Table 2). Against a background of increasing amplitude of beta-activity, there was also a significant decrease in the alpha rhythm by $8.6 \%$ ( $\mathrm{p}<$ 0.01 ). No significant changes were obtained for the rest of the ranges. Within an average of $56.9 \pm 12.9$ seconds after the termination of breath-holding, positivation of the DC potential continued and attained its maximum of $478.7 \pm 101 \mu \mathrm{V}(\mathrm{p}<0.001)$, after which the DC potential level gradually began to recover to its original level. The recovery time was $205.7 \pm 84.0$ seconds on the average.
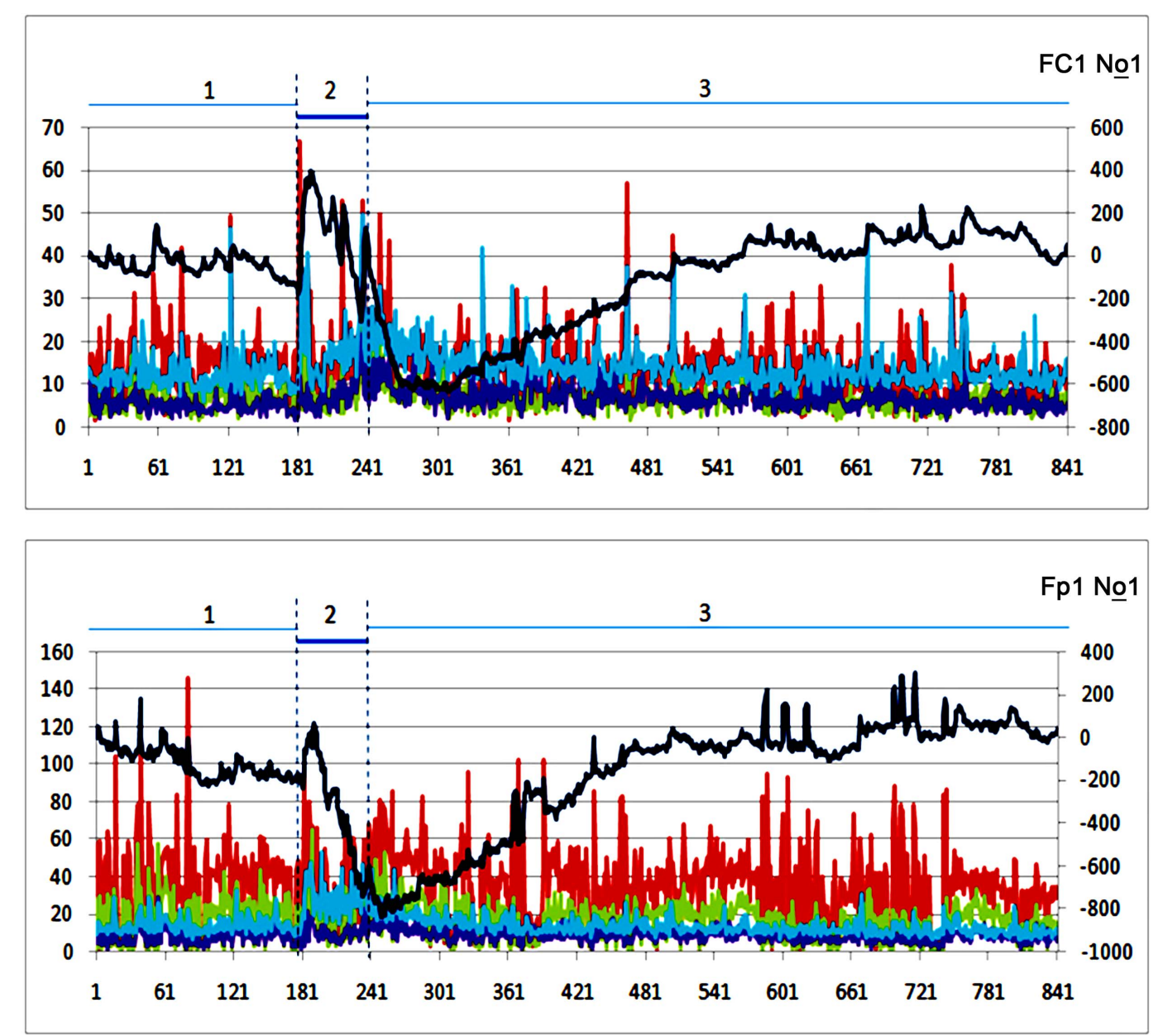

Figure 3. Examples of peculiarities in the changes of the EEG and DC potential before (1), during (2) and after (3) hyperventilation in leads FC1 and Fp1 of subject No. 1. The rest of the designations are the same as in Figure 1. 

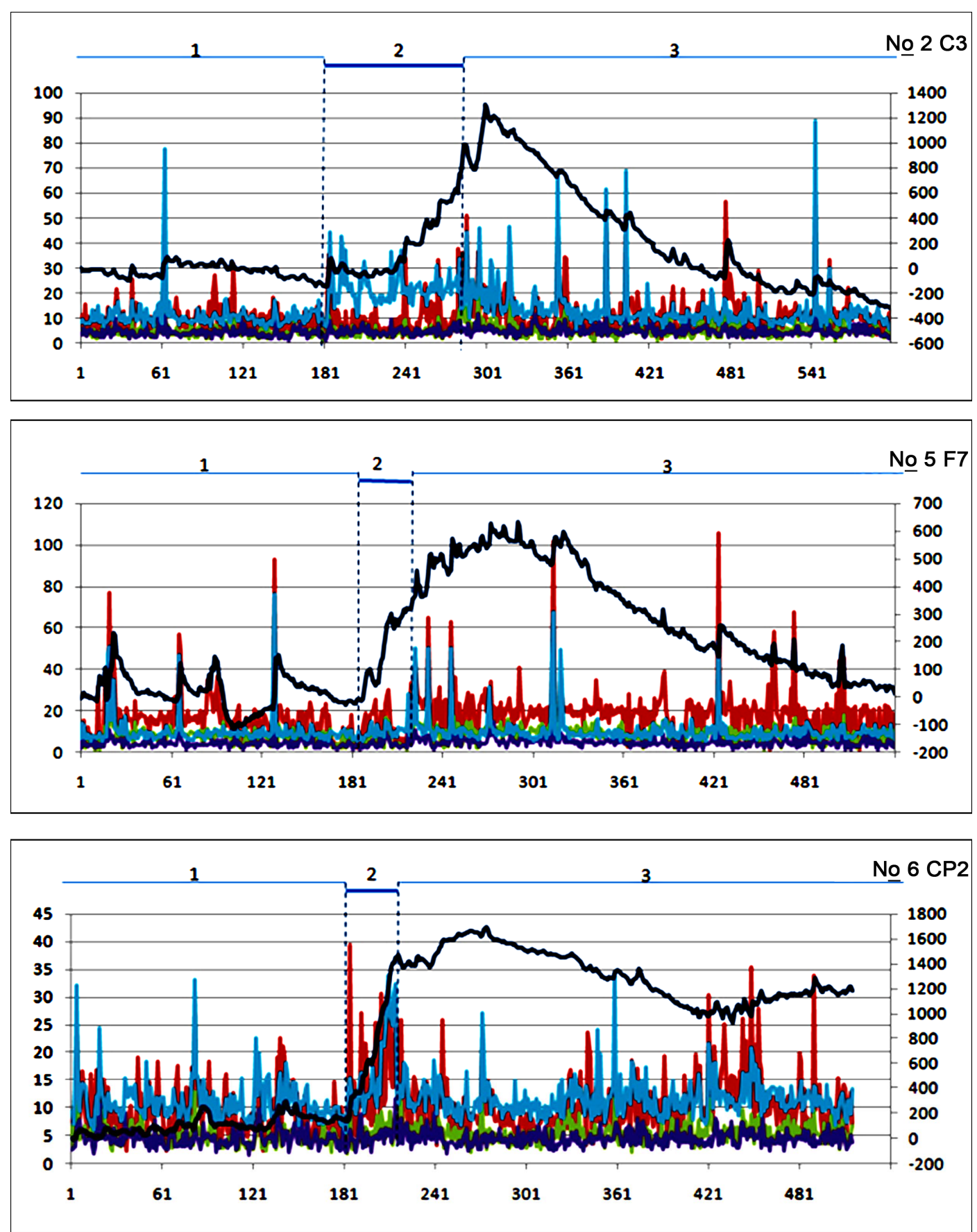

Figure 4. Simultaneous changes in the DC potential and EEG rhythm amplitudes before (1), during (2) and after (3) the breath-holding for subject No. 2 in lead C3, subject No. 5 in lead F7 and subject No. 6 in lead $\mathrm{CP} 2$. The rest of the designations are the same as in Figure 1.

After the termination of hypoventilation, against a background of an even larger positive DC potential shift, there was also a significant increase in the EEG rhythm amplitude in all the ranges as compared to the initial level (see Table 2). The largest increase in the EEG amplitude occurred in the delta range and was as high as $45.6 \%(\mathrm{p}<0.001)$. This EEG feature lasted for at least another 3 minutes after the termination of breath-holding.
Therefore, hypoventilation was accompanied by the positive DC potential shift and differently directed shifts in the beta and alpha ranges of EEG: an amplitude increase in the beta range and activity decrease in the alpha range. After the subjects had stopped holding their breath, positivation of the DC potential continued for about one more minute against a background of increasing EEG amplitude in all ranges. 


\section{DISCUSSION}

Hyperventilation is considered as a model of weak ischemia [29]. Early studies of the influence of hyperventilation on the DC potential and EEG, as a rule were made separately, with only either DC potential $[19,25]$ or EEG [30] recorded. In the studies dealt simultaneously with DC potential and EEG, the DC potential and EEG were recorded during hyperventilation by different electrodes placed some distance apart [14]. In our study, the DC potential and EEG have been recorded in every lead of the same electrode. The obtained results generally correspond to the data already available: during hyperventilation there occurred negativation of the DC potential [14, $19,25,26]$ and increase in EEG rhythm capacity within some ranges, mostly in the slow-wave one [14,30]. Kraaier, V., et al. (1988) also pointed out an increase in slow activity and a decrease in alpha and beta activity.

However, the approach taken-simultaneous recording and analysis of the DC potential and EEG-has also allowed us to reveal some characteristic features. It has been found that the negative DC potential shift and increase in EEG capacity lasted several more tens of minutes after the finish of 1-minute hyperventilation.

Besides, we have found some individual characteristics of the character of DCEEG change during hyperventilation. In particular, it has been stated that in parallel with the negative shift of the DC potential during this test there also may be its positivation occurring prior to the negative DC potential shift (Figure 3). The positive shift might also have occurred some minutes after the finish of hyperventilation. Nobody has ever identified either positivation of the DC potential in the beginning of hyperventilation or trace the positive DC potential shift after hyperventilation test.

We have also revealed front-back gradient of the amplitude of the negative DC potential during hyperventilation and its interhemispheric asymmetry. Earlier [25] it was found that the peak amplitude of the DC potential occurred in the central leads.
Our study has also shown that a satisfactory DCEEG recording is either possible without painful puncturing the skin with a needle under electrodes.

Hypoventilation caused by holding one's breath is used in the study of some diseases, for example, dyspnea [31]. Our study has shown hypoventilation generally associated with the character of DC potential change opposite to that occurred during hyperventilation: so far from stopping immediately after the finish of breath-holding spell, the positivation of the DC potential attained its maximum after some tens of seconds. The study of DC potential during breath-holding was not found emphasized in any reports known, though there are some works concerning hypoxia modeling by the decrease of the oxygen content in the inspired air $[19,25]$. The positive shift of the DC potential was also mentioned therein. The animal experiments have shown that hypercapnia induces hyperpolarization and the positive DC potential shift [4, $32,33]$.

We still adhere to the neuronal nature of the DC potential and suppose that the arguments adduced in support of the alternative hypothesis $[18,19,34]$ in no way exclude the participation of shifts of the neuronal polarization level in the mechanism of the DC shifts.

The physiological mechanism of hypo- and hyperventilation influence on the functional status of neurons is apparently related to cerebral vascular responses to alteration in oxygen and carbon dioxide contents of blood (Figure 5), with $\mathrm{CO}_{2}$ level known as of first importance. The increase in $\mathrm{CO}_{2}$ content causes acidosis, whereas the decrease in $\mathrm{CO}_{2}$ content gives rise to alkalosis and vasoconstriction. Most likely hyperventilation induces hypocapnia $[14,26,28,35]$ causing vasoconstriction and thus development of ischemic state $[29,36]$. It is well known [37] that during nervous tissue ischemia the resting potential of neurons starts to decrease (i.e. depolarization of cellular membrane develops). It is well known that the ischemic depolarization of neurons is associated with a negative DC potential shift [38,39].

Table 2. Average level of the EEG rhythm amplitudes $(\mu \mathrm{V}, \mathrm{m} \pm \mathrm{SEM})$ within 1 minute before start of breath-hold, during breath holding, and within 3 minutes after the termination of breath holding. Designations: \% is a percent of the rhythm amplitude change relative to the mean amplitude 1 minute before the start of hypoventilation taken as $100 \%$; $\mathrm{p}$ is significance $\mathrm{H}_{0}$.

\begin{tabular}{lccccccccccccc}
\hline & \multicolumn{3}{c}{ Delta } & \multicolumn{3}{c}{ Theta } & \multicolumn{3}{c}{ Alpha } & \multicolumn{3}{c}{ Beta } \\
\cline { 2 - 14 } & $\mathrm{m} \pm$ SEM & $\%$ & $\mathrm{p}$ & $\mathrm{m} \pm$ SEM & $\%$ & $\mathrm{p}$ & $\mathrm{m} \pm$ SEM & $\%$ & $\mathrm{p}$ & $\mathrm{m} \pm$ SEM & $\%$ & $\mathrm{p}$ \\
\hline $\begin{array}{l}\text { Within 1 min before } \\
\text { the breath-hold }\end{array}$ & $10.8 \pm 0.4$ & 100 & & $5.3 \pm 0.1$ & 100 & & $4.3 \pm 0.1$ & 100 & & $10.4 \pm 0.2$ & 100 & \\
$\begin{array}{l}\text { During breath-hold } \\
\text { min after }\end{array}$ & $11.0 \pm 0.4$ & 101.6 & $\mathrm{p}>0.05$ & $4.9 \pm 0.1$ & 94 & $\mathrm{p}>0.05$ & $4.0 \pm 0.1$ & 91.4 & $\mathrm{p}<0.01$ & $13.2 \pm 0.4$ & 126.9 & $\mathrm{p}<0.001$ \\
2 min after & $15.7 \pm 0.4$ & 145.6 & $\mathrm{p}<0.001$ & $7.1 \pm 0.2$ & 135.6 & $\mathrm{p}<0.001$ & $5.0 \pm 0.1$ & 114.2 & $\mathrm{p}<0.001$ & $13.7 \pm 0.4$ & 131.8 & $\mathrm{p}<0.001$ \\
3 min after & $13.3 \pm 0.4$ & 123.7 & $\mathrm{p}<0.001$ & $6.4 \pm 0.2$ & 121.4 & $\mathrm{p}<0.001$ & $4.9 \pm 0.1$ & 113.5 & $\mathrm{p}<0.001$ & $11.8 \pm 0.3$ & 113.8 & $\mathrm{p}<0.001$ \\
\hline
\end{tabular}


The observed pattern of simultaneous changes in DC potential and EEG rhythm amplitude during hyperventilation is generally similar to that obtained from the animal model of relatively weak ischemia [21]. Both cases have shown the negative DC potential shift and increase in the EEG rhythm capacity.

Therefore, a negative DC potential shift and changes in the EEG rhythm amplitude during hyperventilation are apparently responsible for an ischemia-induced bad metabolic (vital) and FS and thus depolarization of membrane potential of neurons. An increase in the EEG rhythm amplitude at that time may be indicative of activation of interneuronal impulsation and, generally, the development of catelectrotonus hyperexcitability [1].

During hypoventilation (voluntary breath-hold), the vascular responses most likely have opposite character $[28,30]$. Breath-holding results in a reduction of oxygen and an accumulation of carbon dioxide in the blood stream. Hypoventilation and associated acidosis induce distention of brain vessels, improvement of blood supply and thus improvement of vital and FS of neurons (Figure 5). A positive DC-potential shift occurs in the process.

In this case, the positive DC potential shift due to improved nutrition for brain cells reflects the changes in membrane resting potential opposite to those occurred during ischemic depolarization, i.e. re- and hyperpolarization. In other words, if ischemic-associated phenomena of nervous tissue, unfavorable for the life of cells, and development of bad FS of brain cells are related to depolarization processes and negative DC-potential shift, then the improved nutrition for brain and development of good FS induce opposite changes: re- and hyperpolarization and positivation of the DC potential. Hyperpolarization of the membrane potential and positive DC-potential shift in combination with an increase in the EEG rhythm capacity may also be due to the effect of neuroprotective drag [21] and reward [10,40].

Generally, the method for processing and representing data we involved in the pilot study has shown its prospects for studying specific features of the change in the FS of brain neurons. Using the only characteristic of EEG for estimating the FS of the brain in the tests applied provides a way to find many common features in the character of EEG changes during hyper- and hypoventilation. This is specifically the increase in rhythm amplitude in some of the ranges, especially towards the finish of testing of immediately after it. On this basis, one may relate it to the formation of similar metabolic and FS in the nervous tissue of the brain. However, using an additional characteristic of the DC-potential level suggests difference in vital and FS in the nervous tissue of the brain during this testing. An increase in the EEG amplitude during hyperventilation was followed by the negative DC potential shift whereas its increase during hypoventilation was followed by the positive DC potential shift.

Individual analysis of simultaneous changes in the DC potential and EEG, associated with hyper- and hypoventilation, implies that functional changes occurring in the cerebral space may be more complex than those observed during averaging the leads for the subjects. In particular, the positive DC shift preceding the negative one in separate leads for some of the subjects during hyperventilation suggests the possibility of development of more complex physiological processes than those shown in Figure 5. The possibility of existence of various DCEEG changes under the effect of the same factor with time is considered in S. E. Murik and A. G. Shapkin (2004).

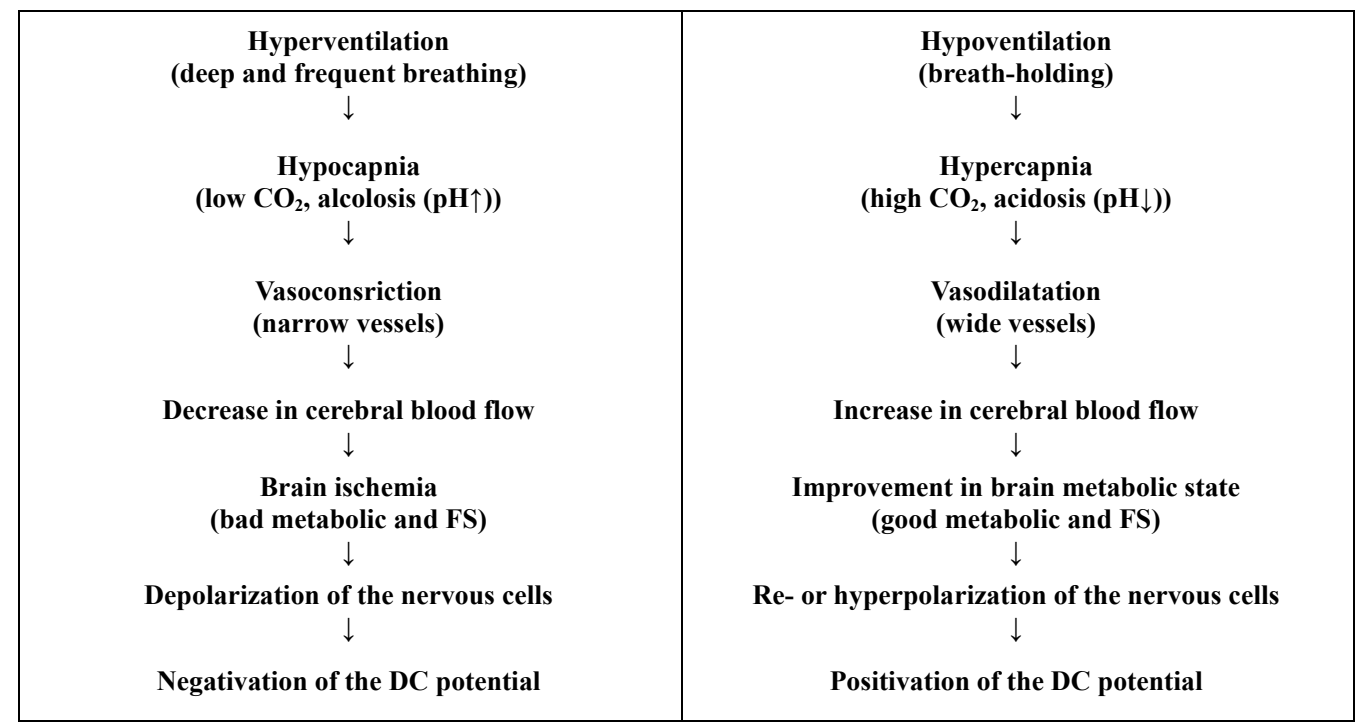

Figure 5. A sample scheme for the processes occurring in the nervous tissue during hyperventilation (1 $\mathrm{min}$ ) and hypoventilation (voluntary breath-hold). 
Generally, the results of the conducted pilot study show the prospects for DCEEG and the method we used for DCEEG data processing to understand the character of functional and metabolic changes in the nervous tissue both in norm and pathology. The method applied for data recording and processing makes multi-electrode DCEEG recording rather easy.

The well-known character of DC shifts and polarization changes in nervous tissue during ischemia [38,39, $41]$, reward $[9,10]$ and effect of neuroprotective drugs $[21,42,43]$ may serve as further evidence for neural rather than any other origin of the DC potential.

We further suggest that the negative DC potential shift and an increase in the EEG rhythms amplitude in cases other than 1-min hyperventilation will also reflect deterioration of metabolic and functional state of brain cells whereas the positive DC potential shift and an increase in the EEG rhythms amplitude (as during voluntary breathhold) will be associated with an inverse process of improving their functional and metabolic (vital) state.

\section{ACKNOWLEDGEMENTS}

The author is grateful to Prof. Walter Paulus (Department of Clinical Neurophysiology, University of Gettingen, Germany) for given possibility and suggesting in making this investigation. The author also expresses his sincere thanks to Vera Moliadze and Olga Lucia Gamboa for their assistance. This work became possible only thank support by grant the European Erasmus Mundus Program (TripleI2009212).

\section{REFERENCES}

[1] Murik, S.E. (2004) Omega electroencephalography (DCEEG) as a new way of estimation the functional and metabolic state of neural tissue. Bulletin of Eastern-Siberian Scientific Center SB RAMS, 3, 189-194.

[2] Vanhatalo, S., Voipio, J. and Kaila, K. (2005) Full-band EEG (FbEEG): An emerging standard in electroencephalography. Clinical Neurophysiology, 116, 1-8. doi:10.1016/j.clinph.2004.09.015

[3] Cowen, M.A. (1974) The brain as generator of transcephalically measured direct current potentials. Psychophysiology, 11, 321-335. doi:10.1111/j.1469-8986.1974.tb00551.x

[4] Caspers, H., Speckmann, E.-J. and Lehmenkiihler, A. (1987) DC potentials of the cerebral cortex seizure activity and changes in gas pressures. Reviews of Physiology, Biochemistry and Pharmacology, 106, 127-178. doi:10.1007/BFb0027576

[5] Birbaumer, N., Elbert, T., Canavan, A.G. and Rockstroh, B. (1990) Slow potentials of the cerebral cortex and behavior. Physiological Reviews, 70, 1-41.

[6] Speckmann, E.-J. and Elger, C. (1999) Introduction to the neurophysiological basis of the EEG and DC potentials. In: Niedermeyer, E.L. and da Silva, F., Eds., Electroencephalography: Basic Principles, Clinical Applications and Related Fields, Williams and Wilkins, Baltimore, 15-27.

[7] Goldring, S. and O'Leary, J.-L. (1951) Summation of certain enduring sequelae of cortical activation in the rabbit. Electroencephalography and Clinical Neurophysiology, 3, 329-340. doi:10.1016/0013-4694(51)90081-8

[8] Rowland, V. and Andersen, R. (1971) Brain steady potential shifts. Progress in Psychobiology and Physiological Psychology, 4, 37-51.

[9] Schmitt, B., Molle, M., Marshall, L., Hallschmid, M. and Born, J. (2001) Scalp recorded direct current (DC) potential shifts associated with food intake in hungry humans Behavioural Brain Research, 119, 85-92. doi:10.1016/S0166-4328(00)00338-7

[10] Marczynski, T.J. (1993) Neurochemical interpretation of cortical slow potentials as they relate to cognitive processes and a parsimonious model of mammalian brain. In: Callum, W.C. and Curry, S.H., Eds., Slow Potential Changes in the Human Brain, Plenum Press, New York, 253275.

[11] Roland, P.E. (2002) Dynamic depolarization fields in the cerebral cortex. Trends in Neurosciences, 25, 183-190. doi:10.1016/S0166-2236(00)02125-1

[12] Speckmann, E.-J., Caspers, H. and EIger, C.E. (1984) Neuronal mechanisms underlying the generation of field potentials. In: Elbert, B., Rockstroh, W., Lutzenberger, N., and Birbaumer, N., Eds., Self-Regulation of the Brain and Behavior, Springer, Berlin, 9-25.

[13] Somjen, G.G. (1973) Electrogenesis of sustained potentials. Progress in Neurobiology, 1, 201-237. doi:10.1016/0301-0082(73)90012-9

[14] Rockstroh, B. (1990) Hyperventilation-induced EEG changes in humans and their modulation by an anticonvulsant drug. Epilepsy Research, 7, 146.

[15] Laming, P.P., Kimelberg, H., Robinson, S., Salm, A., Hawrylak, N., Muller, C., Roots, B. and Ng, K. (2000) Neuronal-glial interacnions and behavior. Neuroscience \& Biobehavioral Reviews, 24, 295-340. doi:10.1016/S0149-7634(99)00080-9

[16] Rebert, C.S. (1978) Electrogenesis of slow potential changes in the central nervous system: A summary of issues. In: Otto, D.A., Ed., Multidisdplinary Perspectives in Event Related Brain Potential Research, US Environmental Protection Agency, Washington DC, 3-11.

[17] Roitbak, A.I., Fanardjian, V.V., Melkonyan, D.S. and Melkonyan, A.A. (1987) Contribution of glia and neurons to the surface-negative potentials of the cerebral cortex during its electrical stimulation. Neuroscience, 20, 1057 1067. doi:10.1016/0306-4522(87)90263-6

[18] Vanhatalo, S., Tallgren, P., Becker, C., Holmes, M.D., Miller, J.W., Kaila, K. and Voipio, J. (2003) Scalp-recorded slow EEG responses generated in response to hemodynamic changes in human brain. Clinical Neurophysiology, 114, 1744-1754. doi:10.1016/S1388-2457(03)00163-9

[19] Voipio, J., Tallgren, P., Heinonen, E., Vanhatalo, S. and Kaila, K. (2003) Millivolt-scale DC shifts in the human scalp EEG: Evidence for a nonneuronal generator. Jour- 
nal of Neurophysiology, 89, 2208-2214. doi:10.1152/jn.00915.2002

[20] Tschirgi, R.D. and Taylor, J.L. (1958) Slowly changing bioelectric potentials associated with the blood-brain barrier. American Journal of Physiology, 195, 7-22.

[21] Murik, S.E. and Shapkin, A.G. (2004) Simultaneous recording of the EEG and direct current (DC) potential makes it possible to assess the functional and metabolic state of the nervous tissue. International Journal of Neuroscience, 114, 921-934. doi:10.1080/00207450490450154

[22] Tallgrena, P., Vanhatalo, S., Kailaa, K. and Voipio, J. (2005) Evaluation of commercially available electrodes and gels for recording of slow EEG potentials. Clinical Neurophysiology, 116, 799-806. doi:10.1016/j.clinph.2004.10.001

[23] Marshall, L., Mölle, M., Schreiber, H., Fehm, H.L. and Born, J. (1994) Scalp recorded direct current potential shifts associated with the transition to sleep in man. Electroencephalography and Clinical Neurophysiology, 91, 346-352. doi:10.1016/0013-4694(94)00195-2

[24] Picton, T.W. and Hillyard, S.A. (1972) Cephalic skin potentials in electroencephalography. Electroencephalography and Clinical Neurophysiology, 33, 419-424. doi:10.1016/0013-4694(72)90122-8

[25] Tomita-Gotoh, S. and Hayashida, Y. (1996) Scalp-recorded direct current potential shifts induced by hypocapnia and hypercapnia in humans. Electroencephalography and Clinical Neurophysiology, 99, 90-97. doi:10.1016/0921-884X(96)95170-X

[26] Biilow, I.V., Elbert, T., Rockstroh, B., Luzenberger, W., Canavan, A. and Birbaumer, N. (1989) Effects of hyperventilation on EEG frequency and slow cortical potentials in relation to an anticonvulsant and epilepsy. Psychophysiology, 3, 147-154.

[27] Abbott, D.F., Opdam, H.I., Briellmann, R.S. and Jackson, G.D. (2005) Brief breath holding may confound functional magnetic resonance imaging studies. Human Brain Mapping, 24, 284-290. doi:10.1002/hbm.20086

[28] Markus, H.S. and Harrison, M.J. (1992) Estimation of cerebrovascular reactivity using transcranial Doppler, including the use of breathholding as the vasodilatory stimulus. Stroke, 23, 668-673. doi:10.1161/01.STR.23.5.668

[29] Kraaier, V., Van Huffelen, A.C. and Wieneke, G.H. (1989) The hyperventilation-induced ischaemia model in human neuropharmacology: Neurophysiological and psychometric studies of aniracetam and 3-OH aniracetam. European Journal of Clinical Pharmacology, 36, 605-611. doi:10.1007/BF00637744

[30] Kraaier, V., Van Huffelen, A.C. and Wieneke, G.H. (1988) Changes in quantitative EEG and blood flow velocity due to standardized hyperventilation: A model of transient ischaemia in young human subjects. Electroencephalography and Clinical Neurophysiology, 70, 377-387. doi:/10.1016/0013-4694(88)90015-6

[31] Nishino, T. (2009) Pathophysiology of dyspnea evaluated by breath-holding test: Studies of furosemide treatment. Respiratory Physiology \& Neurobiology, 167, 20-25. doi:10.1016/j.resp.2008.11.007

[32] Caspers, H. (1993) DC potentials of the brain. In: Haschke, W., Roitbak, A.I. and Speckmann, E.-J., Eds., Slow Potential Changes in the Brain, Birkhfiuser, Boston, 920.

[33] Caspers, H. and Speckmann, E.-J. (1974) Cortical DC shifts associated with changes of gas tensions in blood and tissue. In: Remond, A., Ed., Handbook of Electroencephaiography and Clinical Neurophysiology, Elsevier, Amsterdam, 41-65.

[34] Nita, D.A., Vanhatalo, S., Lafortune, F.D., Voipio, J., Kaila, K. and Amzica, F. (2004) Nonneuronal origin of $\mathrm{CO}_{2}$-related DC EEG shifts: An in vivo study in the cat. Journal of Neurophysiology, 92, 1011-1022. dx.doi:10.1152/jn.00110.2004

[35] Picton, T.W., Pivik, R.T. and Godbout, R. (1979) Scalprecorded DC potential shifts associated with hyperventilation in human subjects. Canadian Journal Neurological Sciences, 6, 380.

[36] Kuroda, S., Houkin, K., Hoshi, Y., Tamura, M., Kazumata, K. and Abe, H. (1996) Cerebral hypoxia after hyperventilation causes "re-build-up" phenomenon and TIA in childhood moyamoya disease: A near-infrared spectroscopy study. Child's Nervous System, 12, 448-453.

[37] Koroleva, V.I. and Vinogradova, L.V. (2000) Ischemic and hypoxic depolarization in the neocortex. Journal of Highest Nerve Activity, 50, 612-621.

[38] Dijkhuizen, R.M., Beekwilder, J.P., van der Worp, H.B., van der Sprenkel, J.W.B., Tulleken, K.A. and Nicolay, K. (1999) Correlation between tissue depolarizations and damage in focal ischemic rat brain. Brain Research, 840, 194-205. doi:10.1016/S0006-8993(99)01769-2

[39] Higuchi, T., Takeda, Y., Hashimoto, M., Nagano, O. and Hirakawa, M. (2002) Dynamic changes in cortical NADH fluorescence and direct current potential in rat focal ischemia: Relationship between propagation of recurrent depolarization and growth of the ischemic core. Journal of Cerebral Blood Flow and Metabolism, 22, 71-79. doi:10.1097/00004647-200201000-00009

[40] Marczynski, T.J., York, J.L. and Hackett, J.T. (1969) Steady potential correlates of positive reinforcement: Reward contingent positive variation. Science, 163, 301.

[41] Kohno, K., Back, T., Hoehn-Berlage, M. and Hossmann, K.A. (1995) A modified rat model of middle cerebral artery thread occlusion under electrophysiological control for magnetic resonance investigations. Magnetic Resonance Imaging, 13, 65-71. doi:10.1016/0730-725X(94)00081-D

[42] Sufianova, G.Z., Murik, S.E., Sufianov, A.A., Usov, L.A., Shapkin, A.G. and Taborov, M.V. (2002) Functional estimation of the cyclopentyladenosine neuroprotective action according to EEG at a focal cerebral ischemia in the rats. Bulletin of Eastern-Siberian Scientific Center SB RAMS, 1, 179-185.

[43] Shefner, S.A. and Chiui, R.H. (1986) Adenosine inhibits locus coereleus neurons: An intracellular study in a rat brain slices preparation. Brain Research, 366, 364-368. doi:10.1016/0006-8993(86)91320-X 